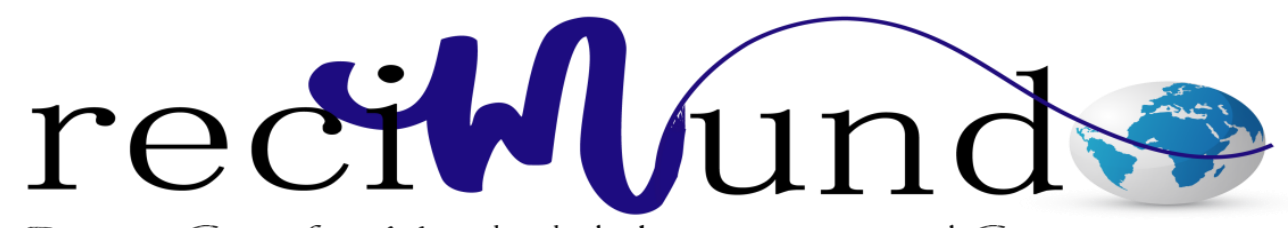

Revista Científica Mundo de la Investígación y el Conocimiento

Jorge Alyghyery Merchán Villamar a; Walter Kiko Salgado Salguero ${ }^{\text {b; }}$ Cinthia Larco Ferrin $^{c}$

Tricomoniasis vaginal. La más común de las Enfermedades de Transmisión Sexual

Revista Científica Mundo de la Investigación y el Conocimiento. Vol. 1 núm., 5, diciembre, 2017, pp. 650-660

DOI: 10.26820/recimundo/1.5.2017.650-660

Editorial Saberes del Conocimiento

a. Universidad de Guayaquil.

b. Universidad de Guayaquil.

c. Universidad de Guayaquil. 


\section{Tricomoniasis vaginal. La más común de las Enfermedades de Transmisión}

Sexual

Vol. 1, núm. 5., (2017)

Jorge Alyghyery Merchán Villamar; Walter Kiko Salgado Salguero; Cinthia Larco Ferrin

\section{RESUMEN}

Trichomonas vaginalis, es un protozoo parásito que infecta el tracto urogenital produciendo la más común de las enfermedades de transmisión sexual. Es una enfermedad que la padecen en un mayor porcentaje las mujeres, sin embargo hay casos probados donde la enfermedad por ser de transmisión sexual puede afectar a los hombres. La metodología utilizada para la presente investigación fue una revisión bibliográfica documental no experimental, ya que se utilizaron múltiples fuentes para obtener información acerca de esta enfermedad. En la mayoría de los trabajos revisados se realizaron estudios a pacientes para determinar el padecimiento o no de la enfermedad y de esta manera generar resultados que generen los mejores tratamientos y mecanismos de prevención en la sociedad. Entre algunos de los resultados se comprueba que es una enfermedad que la padecen en un alto porcentaje las mujeres, el tratamiento más recomendado es la administración por vía oral de metronidazol y entre las recomendaciones más comunes se mencionan disminuir la promiscuidad, la protección con el uso de preservativo, la consulta y tratamiento oportunos de las personas afectadas.

Palabras claves: Tratamiento, transmisión, parasito, enfermedad, sexual. 


\title{
Tricomoniasis vaginal. La más común de las Enfermedades de Transmisión Sexual
}

Vol. 1, núm. 5., (2017)

Jorge Alyghyery Merchán Villamar; Walter Kiko Salgado Salguero; Cinthia Larco Ferrin

\begin{abstract}
Trichomonas vaginalis, is a protozoan parasite that infects the urogenital tract producing the most common sexually transmitted diseases. It is a disease that women suffer in a greater percentage, however there are proven cases where the disease because of being sexually transmitted can affect men. The methodology used for the present investigation was a nonexperimental documentary literature review, since multiple sources were used to obtain information about this disease. In most of the studies reviewed, studies were carried out on patients to determine the condition or not of the disease and in this way generate results that generate the best treatments and prevention mechanisms in society. Among some of the results it is found that it is a disease that women suffer in a high percentage, the most recommended treatment is the oral administration of metronidazole and among the most common recommendations are mentioned decrease promiscuity, protection with the use of condoms, the timely consultation and treatment of the people affected.
\end{abstract}

Keywords: Treatment, transmission, parasite, illness, sexual. 


\section{Tricomoniasis vaginal. La más común de las Enfermedades de Transmisión}

Sexual

Vol. 1, núm. 5., (2017)

Jorge Alyghyery Merchán Villamar; Walter Kiko Salgado Salguero; Cinthia Larco Ferrin

\section{Introducción.}

T. vaginalis fue observada por primera vez por Alfred Donné en 1836 en preparaciones microscópicas de exudados uretrales y vaginales humanas. El nombre de Trichomonas se lo debe a su similitud con los "Tricodes" y las "Monas". La comunidad médica internacional no le creyó al Dr. Donné que este flagelado estuviese involucrado en la producción de patología, hasta que posteriormente Ehrenberg, en 1838, demuestra que el habitat "normal" en el humano es la vagina y por esta razón le da el nombre actual a esta especie parasitaria (Costamagna,2008).

Trichomonas vaginalis, el agente etiológico de la trichomonosis humana, es un protozoo parásito que infecta el tracto urogenital produciendo la más común de las enfermedades de transmisión sexual. Esta parasitosis presenta una distribución cosmopolita y ha sido identificada en personas entre 15 y 50 años de edad en todos los grupos raciales y estratos socioeconómicos (Hernández et al 2009).

La infección por este parásito constituye una de las mayores causas de vaginitis, cervicitis y uretritis en mujeres, y puede causar prostatitis, uretritis y síndromes del tracto genito-urinario bajo en el hombre. Su control es importante, teniendo en cuenta la elevada incidencia de infecciones agudas, complicaciones y secuelas, y el papel que puede jugar como cofactor en la transmisión del virus de la inmunodeficiencia humana (VIH).Además, se plantea que existe asociación de T. vaginalis con otros virus y gérmenes causantes de infecciones de transmisión sexual (ITS) (Hernández et al 2009).

El período de incubación de la infección por $T$. vaginalis es de 4 a 28 días. Durante esta etapa la microbiota de Doderlein (compuesta en su mayoría por Lactobacillus acidophilus) se 


\section{Tricomoniasis vaginal. La más común de las Enfermedades de Transmisión Sexual}

Vol. 1, núm. 5., (2017)

Jorge Alyghyery Merchán Villamar; Walter Kiko Salgado Salguero; Cinthia Larco Ferrin

conserva y hay poca o ninguna reacción inflamatoria. A medida que la infección avanza estos parámetros se invierten, es decir, paulatinamente desaparecen los lactobacilos y se reemplaza por una microbiota bacteriana mixta, con un aumento del número de leucocitos y de parásitos (Hernández et al 2009).

A fines del siglo pasado y en comparación con otras enfermedades infecciosas, la morbilidad atribuible a las enfermedades de transmisión sexual (ETS), experimentó un aumento, por lo que es importante conocer la magnitud y tendencia de cada una de ellas para implementar las medidas de control pertinentes de acuerdo a la realidad epidemiológica (Neira et al 2005).

A pesar de que se tienen datos acerca de la incidencia de esta parasitosis al nivel internacional, la actual prevalencia de trichomoniasis en la población general es desconocida, porque la enfermedad no es notificada, y además, por la sensibilidad relativamente baja del método diagnóstico más empleado, que es el examen directo simple del exudado vaginal (Rojas et al 1999).

El presente estudio se realiza con la finalidad de conocer todo lo relacionado con esta afección tan común en las mujeres, cuáles pueden ser sus efectos negativos, como puede prevenirse y tomar todas las acciones necesarias para disminuir sus consecuencias en la salud de la mujer y el hombre.

\section{Metodología.}

Para el presente estudio se utilizó como metodología una revisión bibliográfica documental no experimental en vista de que realizó una revisión y análisis de diferentes artículos 


\section{Tricomoniasis vaginal. La más común de las Enfermedades de Transmisión}

Sexual

Vol. 1, núm. 5., (2017)

Jorge Alyghyery Merchán Villamar; Walter Kiko Salgado Salguero; Cinthia Larco Ferrin médicos y de opinión referentes a la Trichomonas vaginalis para de esta manera determinar sus orígenes, causas y poder llegar a conclusiones importantes que generen las recomendaciones pertinentes para la prevención y control de esta patología.

\section{Resultados.}

Los síntomas se desarrollan de 4 a 30 días después de la infección; periodos de incubación más cortos se relacionan con una enfermedad más grave. La presencia de síntomas es más frecuente e importante en el sexo femenino. En el hombre provoca escasos o nulos síntomas Las variadas formas clínicas de la enfermedad dependen probablemente del número y virulencia del parásito y de la resistencia del hospedero. Se plantea que los síntomas son mayor cuanto mayor es el número de parásitos y cuanto más alcalino sea el Ph. vaginal. La importancia de conocer el grupo de individuos asintomáticos, radica en que ellos actúan como portadores sanos y pueden transmitir sexualmente el parásito a otras personas, Botero \& Restrepo (1992), son de la opinión de la infección se puede presentar en tres formas: vaginitis asintomática, vulvo vaginitis aguda o crónica y uretritis (Cadena et al 2006).

La presencia de síntomas, como consecuencia de la infección por este parásito, es más frecuente en el sexo femenino. En el hombre, por lo general, la infección transcurre con escasa o nula sintomatología. Se ha señalado que entre 25 y $50 \%$ de las mujeres infectadas permanecieron asintomáticas con un $\mathrm{pH}$ vaginal entre 3,8 y 4,2 y microbiota vaginal normal.De estas, $50 \%$ desarrollaron síntomas clínicos en los 6 meses posteriores. Las variadas formas clínicas de la enfermedad dependen probablemente del número, de la virulencia del parásito y de la resistencia del hospedero (Hernández et al 2009). 


\section{Tricomoniasis vaginal. La más común de las Enfermedades de Transmisión Sexual}

Vol. 1, núm. 5., (2017)

Jorge Alyghyery Merchán Villamar; Walter Kiko Salgado Salguero; Cinthia Larco Ferrin

Al examen ginecológico, el cuello uterino se presenta alterado con aspecto edematoso, eritematoso y friable, con áreas puntiformes de color rojo intenso que se entienden además, a la vulva y vagina, este ha sido considerado el signo clínico más específico para el diagnóstico de la trichomonosis vaginal. El exudado inflamatorio cubre la mucosa vaginal, y la vulvitis suele estar marcada por la presencia de eritema, dolor y edema. También se ha descrito la presencia de erosiones cervicales, las que algunos autores han interpretado como una predisposición al carcinoma de cuello (Hernández et al 2009).

Los tratamientos locales con vinagre, clotrimazol, etcétera, alivian los síntomas, pero la curación sólo se logra con base en la administración de metronidazol por vía oral, siempre administrando tratamiento a la pareja sexual. Esta terapéutica selectiva se explicaría porque la ferredoxina y el bajo potencial redox del parásito anaerobio facilitan la reducción del grupo nitro, formándose los productos intermedios citóxicos y parasiticidas (Carrada-Bravo, 2006).

\section{Recomendaciones.}

Por ser la tricomoniasis una ETS y por lo tanto un problema de salud pública, su enfrentamiento debería realizarse sobre la base de un programa que incluya educación con el objeto de permitir reconocer síntomas considerados normales por el criterio popular ("flores blancas"), disminuir la promiscuidad, la protección con el uso de preservativo, la consulta y tratamiento oportunos de las personas afectadas; acciones que llevarían a aminorar el riesgo, disminuir la transmisión y la evolución de la enfermedad respectivamente (Neira et al 2005).

La importancia del tema, lo contradictorio que resulta en ocasiones y la poca información al respecto, exige continuar profundizando en el estudio de la biología del parásito y su relación 


\section{Tricomoniasis vaginal. La más común de las Enfermedades de Transmisión}

Sexual

Vol. 1, núm. 5., (2017)

Jorge Alyghyery Merchán Villamar; Walter Kiko Salgado Salguero; Cinthia Larco Ferrin con otros patógenos de importancia médica. Este conocimiento sería útil para orientar acciones de salud más efectivas para el control de estos agentes infecciosos (Hernández et al 2009).

Los trastornos que ocasiona la vaginitis por Trichomonas vaginalis es un problema social de importancia, no porque conlleve a casos fatales, sino por lo desagradable de los síntomas que provoca y que repercuten de forma directa en el estado sanitario, la psiquis del paciente e incluso, en la incapacidad temporal para el trabajo. Por otra parte, es una enfermedad de transmisión sexual que si no se diagnostica y cura rápido puede propagarse a otros individuos (Maciques Rodríguez et al 2002).

Los síntomas y signos de la tricomoniasis son inespecíficos. Por otro lado, la coexistencia de la parasitosis con otras enfermedades de transmisión sexual (ETS) es frecuente, por lo que se ha recomendado afinar el diagnóstico mediante examen microscópico directo del exudado «en fresco», de preferencia con técnica de campo oscuro o de contraste de fases, que permiten observar la movilidad característica del protozoario. El frotis teñido con el método de Papanicolaou tiene sensibilidad de 60 a $70 \%$ y con el cultivo podría lograrse $95 \%$ de diagnósticos, pero el costo es mucho mayor y se hace más tardado, hasta 48 horas (CarradaBravo, 2006).

\section{Conclusiones.}

El diagnóstico de certeza de esta parasitosis debe estar basado en algún método de demostración del parásito. El método de examen directo de la secreción vaginal permanece aún como el más ampliamente utilizado, por ser un procedimiento sencillo, rápido y de bajo costo, 


\section{Tricomoniasis vaginal. La más común de las Enfermedades de Transmisión Sexual}

Vol. 1, núm. 5., (2017)

Jorge Alyghyery Merchán Villamar; Walter Kiko Salgado Salguero; Cinthia Larco Ferrin

pero que bajo las mejores circunstancias sólo alcanza una sensibilidad de 50-70 \% (Rojas et al 1999).

La alta sensibilidad del cultivo in vitro permite la recomendación de este proceder fundamentalmente en los casos de sospecha clínica de esta parasitosis donde no se haya podido comprobar por examen directo simple, manteniendo dichos ensayos hasta un tiempo mínimo de 48 h de exposición en medio de cultivo (Rojas et al 1999).

La presencia de altas concentraciones de zinc y otras sustancias inhibitorias en las secreciones prostáticas, ha sido propuesta como un importante mecanismo de defensa del tracto urogenital masculino contra las infecciones por una gran variedad de microorganismos, incluida T. vaginalis; tales sustancias pueden ser importantes para la resolución espontánea de la enfermedad en el hombre (Rojas et al 2002).

Los estudios clínicos y epidemiológicos ofrecen pruebas suficientes acerca del vínculo entre las infecciones de transmisión sexual y la infertilidad, tanto en el hombre como en la mujer. Algunos autores consideran que Trichomonas vaginalis, protozoo flagelado descrito por Donné en 1836, puede ser una causa importante de complicaciones urológicas que son capaces de comprometer la fertilidad (Rojas et al 2002).

En los estudios preclínicos de nuevos productos es necesario realizar ensayos in vitro e in vivo. El método comúnmente empleado para determinar concentración de T. vaginalis es el conteo en cámara de Neubauer. Hay autores que han recurrido a otras técnicas como Kaneda y otros que determinan la concentración de T. vaginalis frente al sulfato de barberina mediante un 


\section{Tricomoniasis vaginal. La más común de las Enfermedades de Transmisión}

Sexual

Vol. 1, núm. 5., (2017)

Jorge Alyghyery Merchán Villamar; Walter Kiko Salgado Salguero; Cinthia Larco Ferrin hematocitómetro, pero no hacen referencia a la correlación del método respecto al conteo en cámara de Neubauer (Meneses et al 2001).

El conocimiento de la verdadera y real prevalencia de Trichomonosis en la población mundial es difícil de precisar, ya que no existen estudios epidemiológicos tranversales que hayan permitido obtener la información necesaria, y además éstos difieren de acuerdo con la sensibilidad del o de los métodos utilizados para el diagnóstico de laboratorio. Algunas estimaciones señalan que el 3,5\% de la población sería portadora del flagelado (Costamagna, 2008).

El diagnóstico por látex además brinda la opción de poder refrigerar o congelar las muestras para ser investigadas con posterioridad, lo cual es imposible realizar cuando se utiliza la microscopia o el cultivo; los resultados son de fácil lectura y se comparan con un control positivo y negativo que descarta cualquier posibilidad de error (Maciques Rodríguez et al 2002).

\section{Bibliografía.}

Cadena, D., Miranda, N., \& Calderón, N. (2006). Tricomoniasis urogenital. Rev Paceña Med Fam, 3(4), 84-9.

Carrada-Bravo, T. (2006). Tricomoniasis vaginal. Rev Mex Patol Clin, 53(3), 151-156.

Costamagna, S. R. (2008). Trichomonas vaginalis. Parasitosis regionales, 139.

Hernández Alvarez, H. M., Sariego Ramos, I., \& Sarracent Pérez, J. (2009). Infección humana por Trichomonas vaginalis y su relación con otros agentes patógenos. Revista Cubana de Obstetricia y Ginecología, 35(4), 108-117.

Maciques Rodríguez, I., \& Alonso Castellanos, M. (2002). Diagnóstico y síntomas clínicos de la trichomoniasis vaginal. Revista Cubana de Obstetricia y Ginecología, 28(2), 0-0.

Meneses Marcel, A., Rojas, L., Sifontes Rodríguez, S., López, Y., \& Sariego Ramos, I. (2001). Aplicación de un método alternativo al conteo en cámara de Neubauer para determinar la 


\section{Tricomoniasis vaginal. La más común de las Enfermedades de Transmisión Sexual}

Vol. 1, núm. 5., (2017)

Jorge Alyghyery Merchán Villamar; Walter Kiko Salgado Salguero; Cinthia Larco Ferrin

concentración de Trichomonas vaginalis. Revista Cubana de Medicina Tropical, 53(3), 180-188.

Neira, O., Correa, L. L., Muñoz, S., Tardío, O., Teresa, M., \& Carabelli, F. (2005). Frecuencia de infección por Trichomonas vaginalis en atención primaria de salud. Revista chilena de obstetricia y ginecología, 70(3), 147-151.

Rojas Rivero, L., Solano Silveira, R., \& Sariego Ramos, I. (1999). Frecuencia de trichomoniasis vaginal en mujeres suspuestamente sanas. Revista Cubana de Higiene y Epidemiología, 37(2), 66-70.

Rojas Rivero, L., Rodríguez Peña, M., Sarría Pérez, C., Palma Monroy, S., Sariego Ramos, I., \& Fraga Nodarse, J. (2002). Frecuencia de infección por Trichomonas vaginalis en parejas con trastornos de la fertilidad. Revista Cubana de Medicina Tropical, 54(2), 85-90. 